\title{
A Control for an Unbalanced 3-Phase Load in UPS Systems
}

\author{
Zbigniew Rymarski, Krzysztof Bernacki, Lukasz Dyga \\ Department of Automatic Control, Electronics and Computer Science, Institute of Electronics, \\ Silesian University of Technology, \\ Akademicka St. 16, 44-100 Gliwice, Poland \\ zbigniew.rymarski@polsl.pl
}

\begin{abstract}
Single input single output systems (SISO), which only control the output voltage of a voltage source inverter (VSI) using multi-loop feedback, are not sufficient in the event of a 3-phase unbalanced load. A much better solution is a Multi Input Single Output (MISO) control. In such a case, not only is the output voltage controlled but also the output current and the output filter inductor current. It seems that using a Passivity Based Control (PBC) is just one of the better solutions for a VSI. The paper presents simulations of a modified Improved PBC (IPBC2) using a stationary $\alpha \beta$ frame in the event of an unbalanced load in a 3-wire delta load. The simulations were verified using the measurements of a 3-phase inverter experimental model with dynamic balanced and unbalanced loads.
\end{abstract}

Index Terms-Passivity based control; Multi input single output control; Unbalanced load; Voltage source inverter.

\section{INTRODUCTION}

The problem of the control of 3-phase inverters in Uninterruptible Power Source (UPS) systems with an unbalanced load is rarely presented in the literature [1]. Single Input Single Output (SISO) systems in which only the output voltages are measured use a multi-loop control [2] with an internal PID-like loop and a repetitive controller in the outer loop (these damp all of the harmonics very effectively) are not able to fulfil the IEEE-519 (output voltage THD $<5 \%$ ) or the EN 62040-3 standard (THD $<8$ $\%$ for better UPS classes) requirements for output filter inductances that are too high [3]. Only the simultaneous control of the output voltages, output currents and output filter inductor currents is an effective solution in damping the distortions of the output voltage, particularly in the case of a standard nonlinear load (rectifier RC load). In recent years, Passivity Based Control (PBC) [4], [5] has been implemented in power systems. The idea of a PBC that only measures the currents (the output voltage was indirectly controlled) was first developed [6]. All of the three state variables are measured and controlled in the Improved PBC (IPBC) [6] or in an Interconnection and Damping

Manuscript received 10 December, 2017; accepted 5 April, 2018.

Krzysztof Bernacki was supported by the Polish National Science Centre, grant no. 2015/17/N/ST7/03720. This work was partially supported by the Polish Ministry of Science and Higher Education funding for statutory activities. The calculations were carried out using the IT infrastructure funded by the GeCONiI project (POIG.02.03.01-24-099/13).
Assignment PBC (IDA-PBC) [7] (based on e.g. [8]). There are other implementations of $\mathrm{PBC}$ in power supply systems, e.g. in the grid connected inverters [9], [10] or in multilevel inverters [11]. However, the important problem of a dynamic unbalanced load in the three-phase systems has not yet been described. The presented paper only concerns one case - a 3-wire, delta unbalanced load (Fig. 1), although it can be further expanded to consider the zero components of voltages and currents [12].

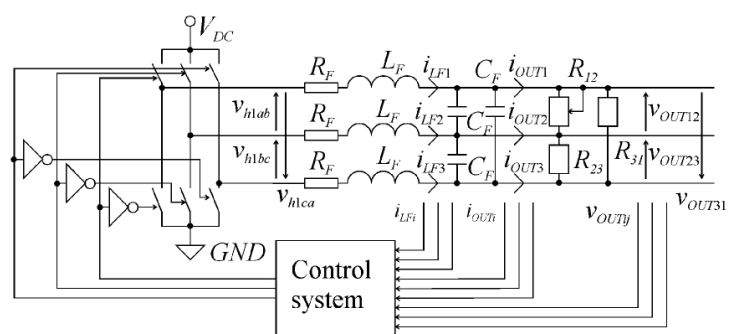

Fig. 1. A two-level 3-phase inverter with an unbalanced 3-wire delta load.

\section{THE CONCEPT OF THE MODIFIED PBC}

The concept of the IPBC that is presented for a single phase inverter in [6] requires that it be transformed into an $\alpha \beta$ stationary frame (Clarke transform). The IDA-PBC [7] permits the decoupling of the error dynamics that is presented in the rotating $d q$ frame, whose advantage is the constant reference values. Decoupling is not necessary in a stationary $\alpha \beta$ frame. The further calculations for $\alpha \beta$ frame will be based on [7] but in this case without the unnecessary decoupling. The results will be similar to an IPBC [6] but the reference filter coil current will be dependent on the $K_{v}$ coefficient (while in [6] it is not dependent). We will call this control IPBC2 because the output voltage error is directly controlled in the control law (15), (16) as in [6] and because of this, its derivative will be in the final control law additionally. For the delta load ( $m_{\alpha \beta}$ is the controlled coefficient-modulation index), we initially assume the state variables vector $\mathbf{x}_{\alpha \beta}$, the control vector $\mathbf{u}_{\alpha \beta}$ and the output vector $\mathbf{y}_{\alpha \beta}$ (the same for $\alpha \beta$ axis):

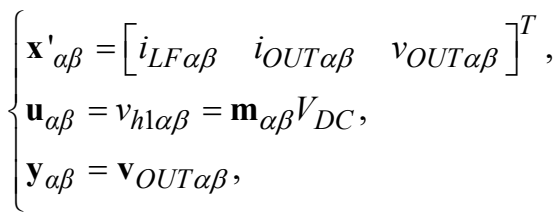




$$
\dot{\mathbf{x}}_{\alpha \beta}^{\prime}=\mathbf{A}_{\alpha \beta} \mathbf{x}_{\alpha \beta}^{\prime}+\mathbf{B}_{\alpha \beta} \mathbf{m}_{\alpha \beta} V_{D C}
$$

For the delta load $C_{F e}=3 C_{F}$, for the star load $C_{F e}=C_{F}$, and $L_{F e}=L_{F}, R_{L F e}=R_{L F}$ in both cases. The state and control matrixes (for one axis $\alpha$ or $\beta$ ) are (3). In further considerations, $i_{\text {OUT } \alpha \beta}$ will be used as the disturbance and (4) the state variables vector $\mathbf{x}_{\alpha \beta}$ will be defined (4) as in [6]. The disturbance variables vector $\mathbf{d}_{\alpha \beta}$ is (6). The disturbance matrix $\mathbf{D}_{\alpha \beta}$ and the damping matrix $\mathbf{R}_{\alpha \beta}$ are (7); the interconnection matrix $\mathbf{J}_{\alpha \beta}$ (for both $\alpha \beta$ axes) and the input (control) variables vectors $\mathbf{m}_{\alpha \beta}$ are (8). The input matrix $\mathbf{G}_{\alpha \beta}$ is (9):

$$
\begin{aligned}
& \mathbf{A}_{\alpha \beta}=\left[\begin{array}{ccc}
-\frac{R_{L F e}}{L_{F e}} & 0 & -\frac{1}{L_{F e}} \\
0 & 0 & 0 \\
\frac{1}{C_{F e}} & -\frac{1}{C_{F e}} & 0
\end{array}\right] \\
& \mathbf{B}_{\alpha \beta}=\left[\begin{array}{c}
1 \\
L_{F e} \\
0 \\
0
\end{array}\right] \\
& \mathbf{x}_{\alpha \beta}=\left[\begin{array}{llll}
L_{F e} i_{L F \alpha} & L_{F e} i_{L F \beta} & C_{F e} v_{O U T \alpha} & C_{F e} v_{\text {OUT } \beta}
\end{array}\right]^{T}= \\
& =\mathbf{P}\left[\begin{array}{llll}
i_{L F \alpha} & i_{L F \beta} & v_{\text {OUT } \alpha} & v_{\text {OUT } \beta}
\end{array}\right]^{T}, \\
& \mathbf{P}=\left[\begin{array}{cccc}
L_{F e} & 0 & 0 & 0 \\
0 & L_{F e} & 0 & 0 \\
0 & 0 & C_{F e} & 0 \\
0 & 0 & 0 & C_{F e}
\end{array}\right], \\
& \mathbf{d}_{\alpha \beta}=\left[\begin{array}{ll}
i_{\text {OUT } \alpha} & i_{O U T \beta}
\end{array}\right]^{T}, \\
& \mathbf{D}_{\alpha \beta}=\left[\begin{array}{cc}
0 & 0 \\
0 & 0 \\
-1 & 0 \\
0 & -1
\end{array}\right] \text {, } \\
& \mathbf{R}_{\alpha \beta}=\left[\begin{array}{cccc}
R_{L F e} & 0 & 0 & 0 \\
0 & R_{L F e} & 0 & 0 \\
-1 & 0 & 0 & 0 \\
0 & -1 & 0 & 0
\end{array}\right] \text {, } \\
& \left\{\begin{aligned}
\mathbf{J}_{\alpha \beta} & =\left[\begin{array}{cccc}
0 & 0 & -1 & 0 \\
0 & 0 & 0 & -1 \\
1 & 0 & 0 & 0 \\
0 & 1 & 0 & 0
\end{array}\right], \\
\mathbf{m}_{\alpha \beta} & =\left[\begin{array}{l}
m_{\alpha} \\
m_{\beta}
\end{array}\right],
\end{aligned}\right. \\
& \mathbf{G}_{\alpha \beta}=\left[\begin{array}{cc}
V_{D C} & 0 \\
0 & V_{D C} \\
0 & 0 \\
0 & 0 \\
0 & 0
\end{array}\right],
\end{aligned}
$$

From (1) to (9), the inverter can be described by (10)

$\dot{\mathbf{x}}_{\alpha \beta}=\left[\mathbf{J}_{\alpha \beta}+\mathbf{R}_{\alpha \beta}\right] \mathbf{P}^{-1} \mathbf{x}_{\alpha \beta}+\mathbf{G}_{\alpha \beta} \mathbf{m}_{\alpha \beta}+\mathbf{D}_{\alpha \beta} \mathbf{d}_{\alpha \beta}$.

The controller is designed to follow the reference state variables of the errors vector

$$
\mathbf{e}_{\alpha \beta}=\mathbf{x}_{\alpha \beta}-\mathbf{x}_{\alpha \beta R E F}
$$

The closed-loop dynamics of the tracking error is described in [7], where the matrix of the gains of the controller $\mathbf{R}_{a \alpha \beta}$ (12) implements the injected $R_{i}$ damping (the gain of the current error) and gain $K_{v}$ of the output voltage error. For $K_{v}=0$, the control law for the $\alpha \beta$ frame will be the same as the one for a conventional PBC [6]. The state space equation for the closed loop inverter [7] is (13). The control law (15) (16) for the stationary $\alpha \beta$ frame is obtained from (14) by subtracting (10) from (13):

$$
\begin{gathered}
\mathbf{R}_{a \alpha \beta}=\left[\begin{array}{cccc}
R_{i} & 0 & 0 & 0 \\
0 & R_{i} & 0 & 0 \\
0 & 0 & K_{v} & 0 \\
0 & 0 & 0 & K_{v}
\end{array}\right] \\
\dot{\mathbf{e}}_{\alpha \beta}=\left[\mathbf{J}_{\alpha \beta}-\left(\mathbf{R}_{\alpha \beta}+\mathbf{R}_{a \alpha \beta}\right)\right] \mathbf{P}^{-1} \mathbf{e}_{\alpha \beta}, \\
\mathbf{G}_{\alpha \beta} \mathbf{m}_{\alpha \beta}=-\left[\mathbf{J}_{\alpha \beta}-\mathbf{R}_{\alpha \beta}\right] \mathbf{P}^{-1} \mathbf{x}_{\alpha \beta R E F}- \\
-\mathbf{R}_{a \alpha \beta} \mathbf{P}^{-1}\left(\mathbf{x}_{\alpha \beta}-\mathbf{x}_{\alpha \beta R E F}\right)-\mathbf{D}_{\alpha \beta} \mathbf{d}_{\alpha \beta}+\dot{\mathbf{x}}_{\alpha \beta R E F}, \\
m_{\alpha \beta} V_{D C}=L_{F e \alpha \beta} d\left(i_{L F \alpha \beta R E F}\right) / d t+R_{L F e} i_{L F \alpha \beta R E F}- \\
-R_{i \alpha \beta}\left(i_{L F \alpha \beta}-i_{L F \alpha \beta R E F}\right)+v_{O U T \alpha \beta R E F}, \\
i_{L F \alpha \beta R E F}=C_{F e} d\left(v_{O U T \alpha \beta R E F}\right) / d t- \\
-K_{v}\left(v_{O U T \alpha \beta}-v_{O U T \alpha \beta R F}\right)+i_{O U T \alpha \beta} .
\end{gathered}
$$

After substituting (16) for (15), there will be a derivative of output voltage in the control law. Therefore, this type of control, which is called an IPBC2, should be faster than the IPBC presented in [6]. The function $H$ of the total energy that is stored in the system is used in all of the types of PBC control [5]. The closed-loop energy function $H\left(\mathbf{x}_{\alpha \beta}, \mathbf{x}_{\alpha \beta R E F}\right)$ for the error vector (17) should have a stable equilibrium at $\boldsymbol{e}_{\alpha \beta}=0$ and is asymptotically stable [12] if (18) and (19):

$$
\begin{aligned}
& H\left(\mathbf{x}_{\alpha \beta}, \mathbf{x}_{\alpha \beta R E F}\right)=\frac{1}{2} \mathbf{e}_{\alpha \beta}^{T} \mathbf{P}^{-1} \mathbf{e}_{\alpha \beta}, \\
& \left.\frac{\partial H\left(\mathbf{x}_{\alpha \beta}, \mathbf{x}_{\alpha \beta R E F}\right)}{\partial \mathbf{x}_{\alpha \beta}}\right|_{\mathbf{x}_{\alpha \beta}=\mathbf{x}_{\alpha \beta R E F}}=0, \\
& \left.\frac{\partial^{2} H\left(\mathbf{x}_{\alpha \beta}, \mathbf{x}_{\alpha \beta R E F}\right)}{\partial \mathbf{x}_{\alpha \beta}^{2}}\right|_{\mathbf{x}_{\alpha \beta}=\mathbf{x}_{\alpha \beta R E F}}>0 .
\end{aligned}
$$

The system is passive if (20). The requirement (20) will be filled for the positively defined $\mathbf{R}_{\alpha \beta}+\mathbf{R}_{a \alpha \beta}$ matrix [7], (21):

$$
\frac{d H\left(\mathbf{x}_{\alpha \beta}, \mathbf{x}_{\alpha \beta R E F}\right)}{d t}<0,
$$




$$
\begin{gathered}
R_{L F e}+R_{i}>0, \\
K_{v}>0 .
\end{gathered}
$$

The other approach that can be used to determine the range of the $R_{i}$ and $K_{v}$ parameters depends on an analysis of the roots of the characteristic polynomial (23) of a closed loop system (13). Roots $\lambda_{1,2}$ (24) should be located in the left-half of the s-plane. It will always be filled for the requirement of $(21),(22)$ :

$$
\begin{aligned}
& \operatorname{det}\left\{\left[\mathbf{J}_{\alpha \beta}-\left(\mathbf{R}_{\alpha \beta}+\mathbf{R}_{\alpha \alpha \beta}\right)\right]-\lambda \mathbf{1}\right\}=0,
\end{aligned}
$$

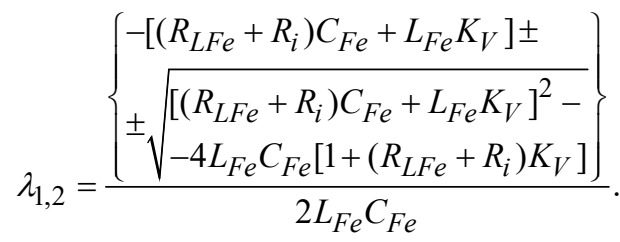

The values of output filter parameters of the simulated and tested inverter model are $L_{F}=3.0 \mathrm{mH}, C_{F}=50 \mu \mathrm{F}$ and $R_{L F}=2 \Omega$. The parameters $L_{F}$ and $R_{L F}$ are the real values for the switching frequency $f_{c}=12800 \mathrm{~Hz}$ of the nominal $L_{F}(50 \mathrm{~Hz})=2.2 \mathrm{mH}$ and the $R_{L F}(\mathrm{DC})=0.2 \Omega$ parameters. This is the result of their fluctuation, which is dependent on the switching frequency, the inductor current and any power losses in the magnetic material at the operating point [13], [14]. In this way the operating point of the inverter influences on its control system [15], [16].

\section{SimUlATION OF THE IMPROVED PBC}

A simulation of the inverter with and without the IPBC2 for the dynamic delta load change $\Delta 43 / \Delta 470 / \Delta 43 \Omega$ and for the dynamic unbalanced delta load should permit the $R_{i}$ and $K_{v}$ values to be selected. In [6], there is a piece of advice that it is better to start with low values (21), (22) and increase them in order to obtain the best results. In the presented simulation, the reasonable low over- and undershoot was reached for $R_{i}=10$ [S] (a too high value of $R_{i}$ damps the output waveform) and $K_{v}=2[1 / \Omega]$ (a too high value of $K_{v}$ causes output voltage oscillations). Figure 2(a) presents the simulation of the output line-to-line voltage $V_{\text {OUT12 }}$ and the line current $I_{O U T 1}$, Fig. 2(b) - the relative voltage overshoot $\left(V_{\text {OUT12- }} V_{\text {OUT12h1 }}\right) / V_{\text {OUT12h } 1 \max } \quad\left(V_{\text {OUT12h1 }}, V_{\text {OUT12h1max }}\right.$ - the calculated fundamental harmonic of $V_{\text {OUT12 }}$ and its amplitude) for the step delta load decrease $(\Delta 43 / \Delta 470 \Omega)$ without the feedback. Figure 2(c) presents the simulation of the output line-to-line voltage $V_{\text {OUT12 }}$ and the line current $I_{\text {OUT1 }}$, Fig. $2(\mathrm{~d})$ - the relative voltage undershoot for the step delta load increase $(\Delta 470 / \Delta 43 \Omega)$ without the feedback.

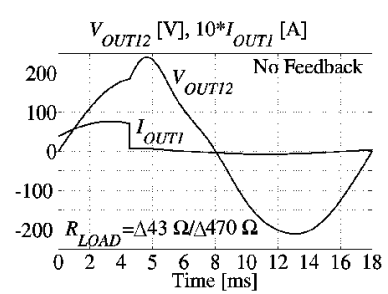

(a)

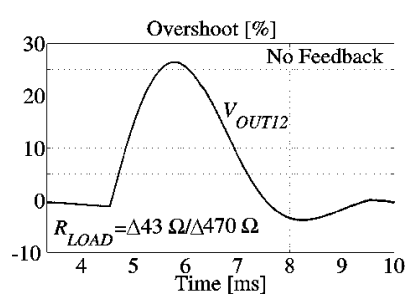

(b)

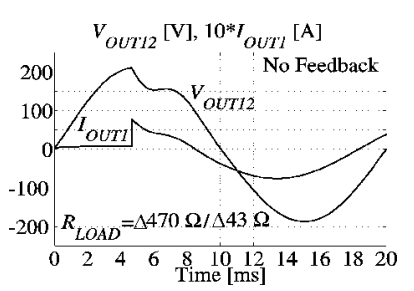

(c)

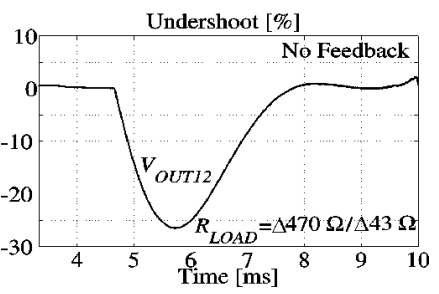

(d)

Fig. 2. The simulated output voltage $V_{O U T 12}$ and current $I_{O U T 1}$ waveforms and voltage VOUT12 over- and undershoot for the inverter without the feedback for the step delta load change $(\Delta 43 / \Delta 470 / \Delta 43 \Omega)$ : a) load decrease $(\Delta 43 / \Delta 470 \Omega)$; b) relative voltage overshoot; c) load increase $(\Delta 470 / \Delta 43 \Omega)$; d) relative voltage undershoot.

Figure 3(a) presents the simulation of the output line-toline voltage $V_{O U T 12}$ and the line current $I_{O U T 1}$, Fig. 3(b) - the relative voltage overshoot for the step delta load decrease $(\Delta 43 / \triangle 470 \Omega$ ) for the IPBC2 with the control law (15), (16) after adjustment the $R_{i}=10[\Omega]$ and $K_{v}=2[1 / \Omega]$ parameters. Figure 3(c) presents the simulation of the output line-to-line voltage $V_{O U T 12}$ and the line current IOUT1, Fig. 3(d) - the relative voltage undershoot for the step delta load increase $(\Delta 470 / \Delta 43 \Omega)$ for the IPBC2.

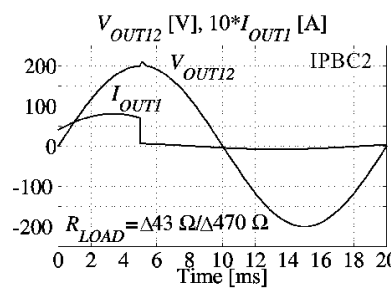

(a)

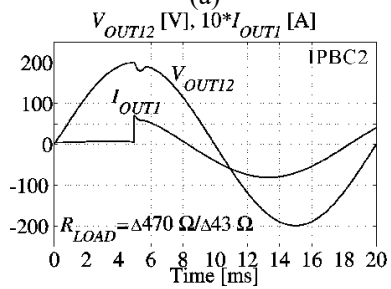

(c)

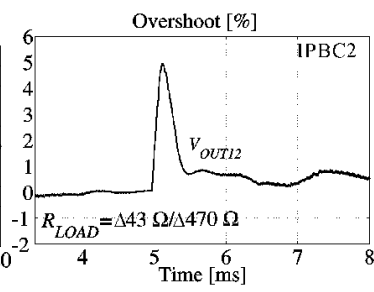

(b)

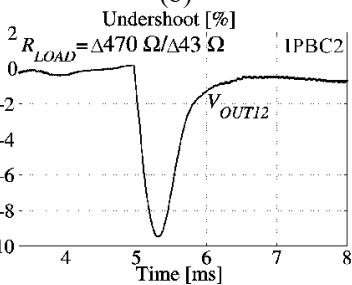

(d)
Fig. 3. The simulated output voltage VOUT12, line current IOUT1 waveforms and voltage VOUT12 over- and undershoot for the inverter with the IPBC2 for the step delta load change $(\Delta 43 / \Delta 470 / \Delta 43 \Omega)$ : a) load decrease $(\Delta 43 / \Delta 470$ $\Omega$ ); b) relative voltage overshoot; c) load increase $(\Delta 470 / \Delta 43 \Omega)$; d) relative voltage undershoot.

Figure 4(a) presents line-to-line voltages and line currents, Fig. 4(b) - relative voltage errors $\left(V_{\text {OUTij }}-V_{\text {OUTijh } 1}\right) / V_{\text {OUTij } h 1 \max }$ for the dynamic balanced delta load $\Delta 47 \Omega$ to the unbalanced delta load $R_{12}=\infty, R_{23}=47 \Omega, R_{31}=47 \Omega$ change for the inverter without the feedback.

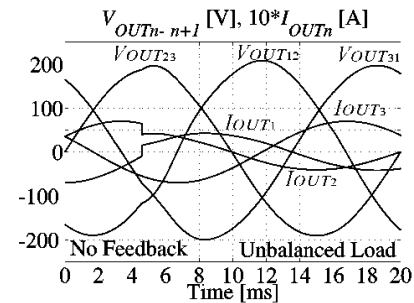

(a)

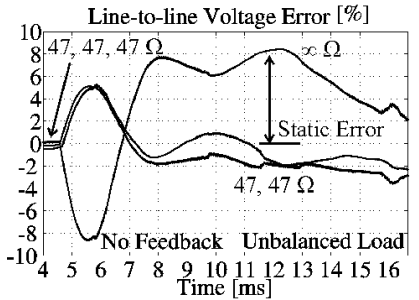

(b)
Fig. 4. The simulated output line-to-line voltages, line current waveform and voltage errors for the inverter without the feedback for the step balanced load $\Delta 47 \Omega$ to the delta unbalanced load $\Delta(\infty-47-47) \Omega$ change: a) balanced to unbalanced load; b) relative voltage errors. 
Figure 5(a) presents the line-to-line voltages and line currents, Fig. 5(b) - relative voltage errors for the IPBC2 for the dynamic balanced delta load $\Delta 47 \Omega$ to the unbalanced delta load $R_{12}=\infty, R_{23}=47 \Omega, R_{31}=47 \Omega$ change. The presented simulations show that the IPBC2 can decrease (45 times) the error of the output line-to-line voltages very efficiently in the event of disturbances in the static or dynamic line currents (balanced or unbalanced static and dynamic load change) for the experimentally adjusted gains of the IPBC2 controller.

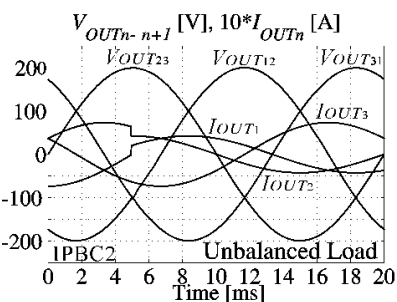

(a)

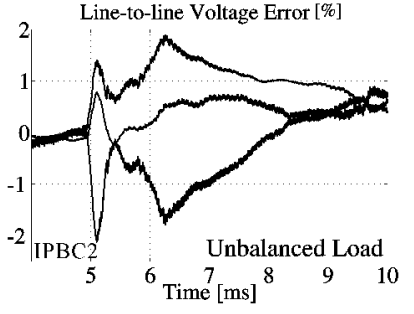

(b)
Fig. 5. The simulated output line-to-line voltages and current waveform and voltage errors for the inverter with the IPBC2 for the step balanced load $\Delta 47 \Omega$ to the delta unbalanced load $\Delta(\infty-47-47) \Omega$ change: a) balanced to unbalanced load; b) relative voltage errors

\section{MEASUREMENTS OF THE EXPERIMENTAL MODEL}

The difference version of the control law (15), (16) was implemented in the experimental model of a 3-level, 3-phase inverter (Fig. 6). The control law was programmed into the STM32F407VG $168 \mathrm{MHz}$ microprocessor. All of the measurements and further floating point calculations were performed during the interrupt procedures. The switching frequency was $f_{c}=12800 \mathrm{~Hz}$.

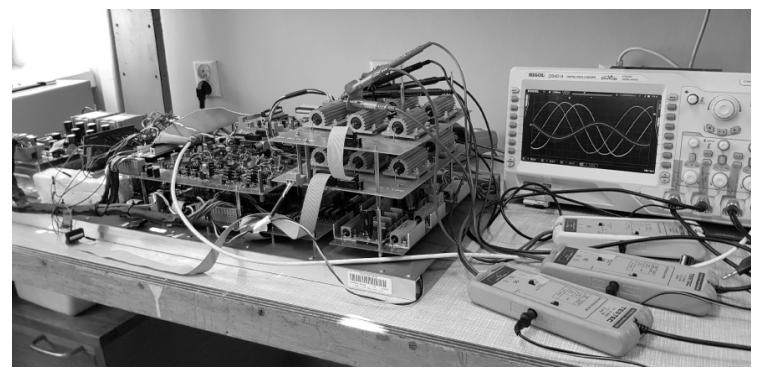

Fig. 6. The experimental model of the 3 -phase inverter.

Figure 7(a) presents the measurements of the output lineto-line voltage $V_{O U T 12}$ and the IOUT1 current for the double step delta load change $(\Delta 43 / \Delta 470 / \Delta 43 \Omega)$, Fig. $7(\mathrm{~b})$ the relative voltage overshoot without the feedback.

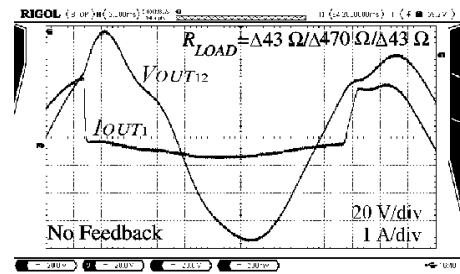

(a)

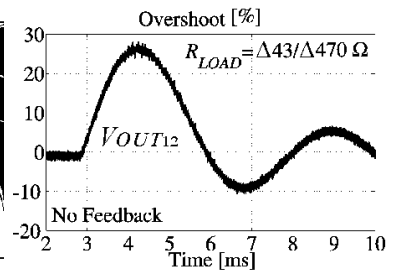

(b)
Fig. 7. The measured output voltage $V_{O U T 12}$, line current IOUT1 waveforms and the voltage $V_{\text {OUT12 }}$ overshoot for the inverter without the feedback for the step delta load change $(\Delta 43 / \Delta 470 / \Delta 43 \quad \Omega)$ : a) step load decrease/increase; b) relative voltage overshoot.

Figure 8(a) presents the measured line-to-line voltages and output currents, Fig. 8(b) their relative errors in the case of the step unbalanced delta load $\Delta(43-470-470) \Omega$ to the balanced delta load $\Delta(470-470-470) \Omega$ change without the feedback. The IPBC2 parameters were experimentally adjusted to $R_{i}=7, K_{v}=0.5$. The higher values of $R_{i}$ damped the output voltage waveforms, while the higher value of $K_{v}$ caused their oscillations.

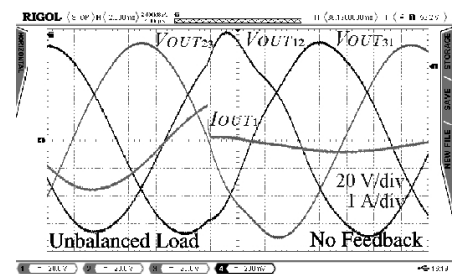

(a)

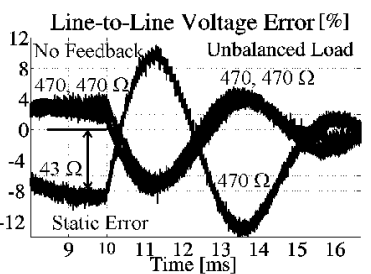

(b)
Fig. 8. The measured output line-to-line voltages, line current IOUT1 waveforms and voltages error for the inverter without the feedback for the step unbalanced delta load 43-470-470 $\Omega$ to the delta balanced load $\Delta 470 \Omega$ change: a) unbalanced to balanced load; b) relative voltage errors.

Three $\mathrm{kHz}$ low pass filters $\left(f_{c}=12.8 \mathrm{kHz}\right)$ were used in the measurement channels. Figure 9(a) presents the measurements of the voltage $V_{O U T 12}$ and the IOUT1 current, Fig. 9(b) - the relative voltage error for the step delta load decrease $(\Delta 43 / \Delta 470 \Omega)$ for the IPBC2. Figure 10(a) presents the measured line-to-line voltages and the line current $I_{O U T 1}$, Fig. 10(b) - their relative errors in case of the step unbalanced delta load $\Delta(43-470-470) \Omega$ to the balanced delta load $\triangle 470 \Omega$ change for the IPBC2. It is very important to adjust the proper modulation ratio $M$ for the effective control of the dynamic load currents that are treated as disturbances. The highest possible $M$ (close to unity) should be used but a better control without the saturation effect will be obtained for a lower value of $M[2]$.

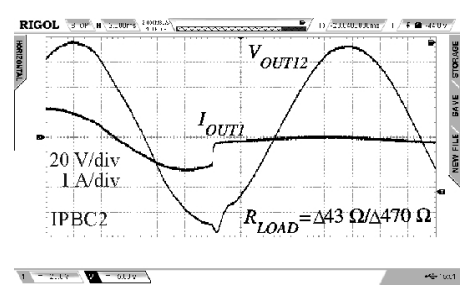

(a)

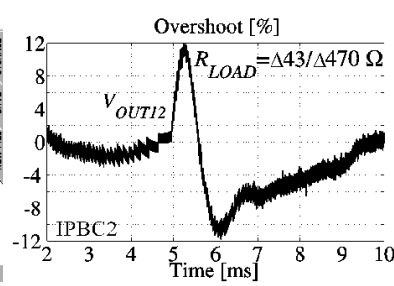

(b)
Fig. 9. The measured output voltage VOUT12, line current IOUT1 waveforms and the voltage VOUT12 overshoot for the inverter with the PBC2 for the step delta load change $(\Delta 43 / \Delta 470 \Omega)$ : a) load decrease $(\Delta 43 / \Delta 470 \Omega)$; b) relative voltage overshoot.

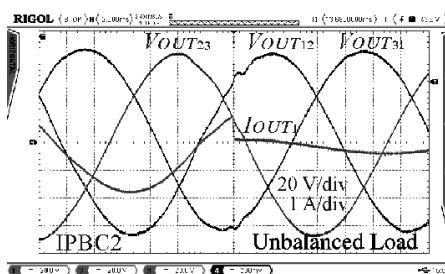

(a)

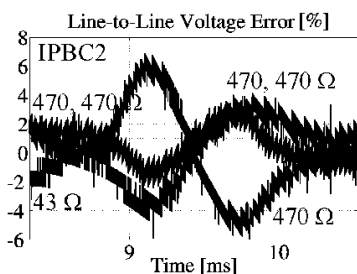

(b)
Fig. 10. The measured output line-to-line voltages, line current IOUT waveform and voltage errors for the inverter with the $\mathrm{PBC} 2$ for the step unbalanced delta load $\Delta(43-470-470) \Omega$ to the delta balanced load $\Delta 470 \Omega$ change: a) unbalanced to balanced load; b) relative voltage errors.

Figure 11(a) presents the simulated and Fig. 11(b) measured control waveforms for the $\alpha$ axis with the existing saturation levels of the PWM at \pm 1 in the simulation and at \pm 3280 in the experimental model for the dynamic delta load change $(\Delta 43 / \Delta 470 \Omega)$. 


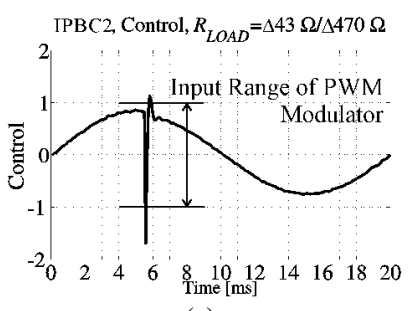

(a)

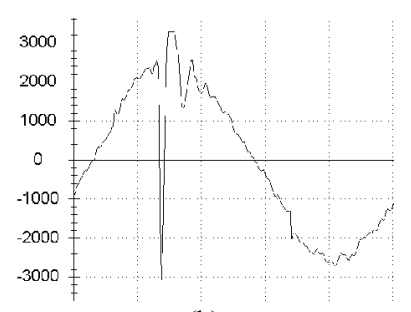

(b)
Fig. 11. The simulated (a) and measured (b)(in units of the PWM) control waveform for the $\alpha$ axis for a decrease in the step delta load $(\Delta 43 / \Delta 470 \Omega)$ : a) simulation of the $\alpha$ axis control; b) measurement of the $\alpha$ axis ctrl.

The modulator saturation will degrade the results of the control process but further decreasing the modulation index $M$ will lead to an output voltage that is too low. The measured results of the implemented IPBC2 vs the inverter without feedback show a reduction in the static error, decreasing the amplitude of the overshoot and shortening the time to set up the output voltage.

\section{DISCUSSION}

The idea of the IDA-PBC for the 3-phase inverter control in the rotating $d q$ frame presented in [7] was based on [8] and it has background in [17]. This paper presents the concept of a PBC in a stationary $\alpha \beta$ frame that reduces the number of calculations compared to the $d q$ frame and no interconnections between the axes occur. The idea of rejecting the interconnections is unnecessary in the stationary $\alpha \beta$ frame. What is more the received control law is similar to the idea of IPBC presented in [6] for the singlephase inverter control. However, the obtained additional derivative of the output voltage error in the final control law improves the transient response of the inverter. And that is why the name of the presented control - IPBC2. The PBC control is very popular in the different applications of the inverters control (e.g. [6], [7], [9]-[11]) but none of them consider the main subject of the paper - the control of the inverter with the unbalanced 3-phase load. The presented controller design takes in care the real values of the inverter parameters measured by authors [13]-[16] what was not showed in the other referenced papers.

\section{CONCLUSIONS}

The presented control in a stationary $\alpha \beta$ frame, which is called IPBC2, works efficiently in the case of a static and dynamic unbalanced 3-wire delta load in a 3-phase inverter. It is important that in the presented case using the stationary $\alpha \beta$ frame, with the reduced number of calculations, the results are similar to the more complex control in the rotating $d q$ frame. The problem of the $\mathrm{PBC}$ in the in the 3phase inverter with the unbalanced load was not presented previously. The idea of adjusting gains of the controller for the balanced dynamic loads and using these values for the static and dynamic unbalanced load was checked successfully in Matlab/Simulink simulations and measurements of the experimental model. The necessary compromise between a sufficient level of the output voltage and the saturation in the PWM modulator is also presented showing that the best result of the control is for the low value of the modulation index which is not acceptable in the customer devoted device. In addition, the problem of the real values of the inverter parameters depending on the operation point and the switching frequency was discussed. The paper presents the theory, software simulations and finally measurements of the experimental model. The convergence of the measurements and simulations is satisfactory, however the PBC controller gains assumed for simulations should be decreased in the experimental model. It can be a result of the inaccurate scaling measured voltages and currents.

\section{REFERENCES}

[1] A. Kawamura, T. Yokoyama, "Comparison of five different approaches for real time digital feedback control of PWM inverters", The 1990 IEEE Industry Applications Society Annual Meeting, 1990, vol. 2, pp. 1005-1011. DOI: 10.1109/IAS.1990.152307.

[2] Z. Rymarski, "The analysis of the output voltage distortions minimization in the 3-phase VSI for the nonlinear rectifier $\mathrm{R}_{\mathrm{O}} \mathrm{C}_{0}$ load", Przeglad Elektrotechniczny, vol. 85, no. 4, pp. 127-132, 2009.

[3] Z. Rymarski, "The discrete model of the power stage of the voltage source inverter for UPS", International Journal of Electronics, vol. 98 , no. 10, 2011, pp. 1291-1304, DOI: 10.1080/00207217.2011.589736.

[4] M. Torres, R. Ortega, "Feedback linerization, integrator backstepping and passivity-based controller designs: a comparision example", Normand-Cyrot D. (Ed.), Perspectives in Control. Theory and Applications, pp. 96-114, 1998. DOI: 10.1007/978-1-4471-1276-1.

[5] D. Hill, J. Zhao, R. Gregg, R. Ortega, "20 years of passivity-based control (PBC): theory and applications", CDC Workshop, Shanghai, PRC, 2009.

[6] H. Komurcugil, "Improved passivity-based control method and its robustness analysis for single-phase uninterruptible power supply inverters", IET Power Electron., vol. 8, no. 8, pp. 1558-1570, 2015. DOI: 10.1049/iet-pel.2014.0706.

[7] F. M. Serra, C. H. De Angelo, D. G. Forchetti, "IDA-PBC control of a DC-AC converter for sinusoidal three-phase voltage generation", International Journal of Electronics, vol. 104, no. 1, pp. 93-110, 2017. DOI: 10.1080/00207217.2016.1191087.

[8] R. Ortega, E. Garcia-Canseco, "Interconnection and damping assignment passivity-based control: a survey", European journal of control, pp. 432-450, 2004. DOI: 10.3166/ejc.10.432-450.

[9] D. Biel, J. M. A. Scherpen, "Passivity-based control of active and reactive power in single-phase $\mathrm{PV}$ inverters", IEEE 26th Int. Symposium on Industrial Electronics (ISIE 2017), Edinburgh, UK, 2017, pp. 999-1004. DOI: 10.1109/ISIE.2017.8001382.

[10] A. F. Cupertino, L. P. Carlette, F. Perez, J. T. Resende, S. I. Seleme, H. A. Pereira, "Use of control based on passivity to mitigate the harmonic distortion level of inverters", IEEE PES Conf. Innovative Smart Grid Technologies (ISGT Latin America 2013), Sao Paulo, Brazil, 2013. DOI: 10.1109/ISGT-LA.2013.6554439.

[11] Zhi Zhang, Na Yao, Caishen Wang, Li Kang, Longyun Kang, "A passivity-based control method for the single-phase three-level inverter", 18th Int. Conf. Electrical Machines and Systems (ICEMS 2015), Pattaya, Thailand, 2015, pp. 1515-1518. DOI: 10.1109/ICEMS.2015.7385281.

[12] H. Akagi, E. H. Watanabe, M. Aredes, "Instanteneous power theory and application to power conditioning", IEEE Press Series on Power Engineering, 2017. DOI: 10.1002/9781119307181.

[13] K. Bernacki, Z. Rymarski, L. Dyga, "Selecting the coil core powder material for the output filter of a voltage source inverter", 20th Electronics Letters, vol. 53, no. 15, pp. 1068-1069, 2017. DOI: 10.1049/el.2017.1534.

[14] Z. Rymarski, "Measuring the real parameters of single-phase voltage source inverters for UPS systems", Int. J. Electron., vol. 104, no. 6, pp. 1020-1033, 2017. DOI: 10.1080/00207217.2017.1279232.

[15] Z. Rymarski, K. Bernacki, L. Dyga, 'The influence of the properties of magnetic materials on a voltage source inverter control", 23rd IEEE Conf. Control Applications (CCA 2014), Antibes, 2014, pp. 1127-1132. DOI: 10.1109/CCA.2014.6981480.

[16] Z. Rymarski, K. Bernacki, "Different approaches to modelling singlephase voltage source inverters for uninterruptible power supply systems", IET Power Electronics, vol. 9, no. 7, pp. 1513-1520, 2016. DOI: $10.1049 /$ iet-pel.2015.0142.

[17] Z. Wang, P. Goldsmith, "Modified energy-balancing-based control for the tracking problem", IET Control Theory \& Applications, vol. 2, no. 4, pp. 310-312, 2008. DOI: 10.1049/iet-cta:20070124. 